\title{
SYSTEMATIC REVIEW OPEN Diagnostic and predictive value of Doppler ultrasound for evaluation of the brain circulation in preterm infants: a systematic review
}

\author{
Fleur A. Camfferman ${ }^{1}$, Robbin de Goederen ${ }^{2}$, Paul Govaert ${ }^{1,3,4,5}$, Jeroen Dudink ${ }^{3,6}$, Frank van Bel ${ }^{6}$, Adelina Pellicer ${ }^{7}$ and \\ Filip Cools ${ }^{1}$ on behalf of the eurUS.brain group
}

INTRODUCTION: Very and extremely preterm infants frequently have brain injury-related long-term neurodevelopmental problems. Altered perfusion, for example, seen in the context of a hemodynamically significant patent ductus arteriosus (PDA), has been linked to injury of the immature brain. However, a direct relation with outcome has not been reviewed systematically. METHODS: A systematic review was conducted to provide an overview of the value of different cerebral arterial blood flow parameters assessed by Doppler ultrasound, in relation to brain injury, to predict long-term neurodevelopmental outcome in preterm infants.

RESULTS: In total, 23 studies were included. Because of heterogeneity of studies, a meta-analysis of results was not possible. All included studies on resistance index (RI) showed significantly higher values in subjects with a hemodynamically significant PDA. However, absolute differences in RI values were small. Studies using Doppler parameters to predict brain injury and long-term neurodevelopmental outcome were inconsistent.

DISCUSSION: There is no clear evidence to support the routine determination of RI or other Doppler parameters in the cerebral arteries to predict brain injury and long-term neurodevelopmental outcome in the preterm infant. However, there is evidence that elevated RI can point to the presence of a hemodynamically significant PDA.

Pediatric Research (2020) 87:50-58; https://doi.org/10.1038/s41390-020-0777-x

\section{INTRODUCTION}

Acquired brain injury is common in the perinatal period. Many extremely and very preterm infants have injury-related long-term neurodevelopmental problems. ${ }^{1}$ Extremes and fluctuations in brain perfusion have been linked to germinal matrix hemorrhage, intraventricular hemorrhage (IVH), and white matter injury. ${ }^{2-5}$ In the adult brain, perfusion is controlled through cerebral autoregulation, the intrinsic capacity of cerebral vessels to adjust muscle tone in order to keep flow more or less constant over a range of blood pressures. ${ }^{6}$ Several studies demonstrated that cerebral autoregulation might not be fully developed in preterm infants, especially in the lower range of gestational ages. Consequently, extremes of systemic perfusion are transmitted unaltered to brain tissue. ${ }^{7}$ Low systemic blood pressure is thought to play an etiological role in the development of IVH in preterm infants. ${ }^{8-10}$ There are studies showing that maintaining blood pressure within normal ranges can prevent severe $\mathrm{IVH}^{5}$ However, there are no clear data demonstrating that this would improve long-term outcome in preterm infants. ${ }^{11,12}$ Low systemic blood flow is associated with IVH with impaired cerebral autoregulation. ${ }^{13}$ In addition, impaired autoregulation is correlated with adverse outcome in infants. ${ }^{14,15}$
In 1979, Bada et al. first reported that cerebral Doppler studies played a role in the diagnosis of neonatal intracranial injury. ${ }^{16,17}$ Doppler provides a valuable tool to follow changes in flow velocities, to assess changes in cerebrovascular resistance and to determine the lower limits of cerebral blood flow autoregulation. $^{18,19}$ For quantitative measurement of flow in a specific vessel, however, knowledge of the internal vascular diameter is necessary, which has proven to be inaccurate in small vessels. ${ }^{20}$ In addition, the angle of insonation between the vessel and the Doppler ultrasound beam has to be as small as possible to prevent inaccuracy in calculating the Doppler shift. To bypass these challenges, many different indices were developed to approximate flow velocity as an estimate of flow, like pulsatility index (PI) and the resistance index (RI). However, the relation of Doppler indices in preterm infants with brain injury and outcome remains controversial. In term birth asphyxia, for instance, low $\mathrm{RI}$ in the anterior cerebral artery has been associated with an adverse neurodevelopmental outcome, ${ }^{21}$ although a relation with extent of specific regional injury on magnetic resonance imaging (MRI) is not documented. In other pathologies, like hydrocephalus, the role of Doppler ultrasound in diagnosis and prognosis is less clear. $^{22,23}$

${ }^{1}$ Department of Neonatology, Universitair Ziekenhuis Brussel, Vrije Universiteit Brussel, Brussels, Belgium; ${ }^{2}$ Dutch Craniofacial Centre Rotterdam, Department of Plastic and Reconstructive Surgery, Erasmus Medical Center University, Rotterdam, The Netherlands; ${ }^{3}$ Department of Neonatology, Erasmus Medical Center University, Sophia Children's Hospital, Rotterdam, The Netherlands; ${ }^{4}$ Department of Neonatology, ZNA Middelheim, Antwerp, Belgium; ${ }^{5}$ Department of Rehabilitation and Physical Therapy, Gent University Hospital, Gent, Belgium; ${ }^{6}$ Department of Neonatology, University Medical Center Utrecht, Wilhelmina Children's Hospital, Utrecht, The Netherlands and ${ }^{7}$ Department of Neonatology, La Paz University Hospital, Madrid, Spain

Correspondence: Fleur A. Camfferman (Fleur.Camfferman@uzbrussel.be)

Members of eurUS.brain are listed at the end of the paper. 
In the present systematic review, we study the value of various Doppler-derived variables in cerebral arteries to predict intracranial injury and long-term neurodevelopmental outcome in the preterm infant. Our search also focused on the relation between cerebral arterial Doppler parameters and patent ductus arteriosus (PDA), since a hemodynamically significant PDA is thought to be one of the determinants of altered brain perfusion patterns in the preterm infant.

\section{METHODS}

Search strategy

Electronic literature searches were performed in MEDLINE, EMBASE, and the Cochrane Central register of Controlled Trials. Full MEDLINE search was first performed on 08-07-2015 using the following Mesh terms: "Infant, Newborn", "Echoencephalography", "Ultrasonography, Doppler", "Blood Circulation Time", "Brain", "Blood Vessels", "Microcirculation", "Cerebral Arteries", "Cerebral Veins", "Blood Flow Velocity", and "Cerebrovascular Circulation". Appropriate text words were added as well. For the full MEDLINE search, see Table 1. Search was restricted to human studies. There was no restriction in publication date. The search was double-checked by the Erasmus University Rotterdam librarian. A weekly current awareness alert in MEDLINE was set, and last update of the search was performed April 2018. In Cochrane, last search was performed April 2018; last EMBASE search was performed may 2015. We looked for additional studies in the reference lists of the studies identified. In addition, we screened some reference suggestions provided by two of the authors, both experts in the field of cranial ultrasound in the newborn (P.G. and A.P.).

Study selection

All experimental as well as observational studies in which Doppler technique was used to assess cerebral perfusion in preterm infants (gestational age $<37$ weeks) and related to mortality and morbidity (IVH, periventricular leukomalacia (PVL), and PDA) during the intensive case period or related to longterm morbidity were included. For observational studies, both prospective cohort and case-control studies were eligible for inclusion. Case reports and case series, defined as $\leq 10$ patients in the study and/or $\leq 5$ patients per outcome group, as well as narrative reviews were excluded. Also, studies focusing on intervention (e.g., cerebral blood flow before and after duct ligation), only describing normative values of a cerebral Doppler index, or in which the main subject was asphyxia or hydrocephalus (not meeting the review question) were not considered eligible for inclusion. Only articles in English, German, French, or Dutch were included. Two review authors (P.G. and F.A.C.) independently assessed eligibility for inclusion of the identified studies based on abstract. Any disagreement was solved through discussion. Subsequently, R.d.G. and F.A.C. independently performed assessment of full-text articles. Disagreement was solved through discussion.
Data collection and data extraction

The methodology for data collection and analysis was based on the Cochrane Handbook of Systematic Reviews of Interventions. ${ }^{24}$ Data extraction was performed independently by two authors (R.d.G. and F.A.C.) using a pre-designed data collection form. Discrepancies in data extraction were resolved by discussion. This extraction form included (I) information on Doppler technique, insonated vessel(s), and Doppler parameter(s) (i.e., Rl, PI, mean velocity, enddiastolic velocity, peak systolic velocity, time averaged velocity, etc.); (II) basic patient characteristics; (III) correlation with systemic parameters; (IV) correlation with pathology during neonatal intensive care unit (NICU) stay; and (V) correlation with longterm outcome. For clarity purposes, we used consistent terminology when describing Doppler indices. Rl, also called Pourcelot index or resistency index, was used for the index described by the following equation: $\mathrm{Rl}=\frac{\mathrm{Vs}_{s}-\mathrm{Vd}_{\mathrm{d}}}{\mathrm{V}_{\mathrm{s}}}$. In this equation, $\mathrm{Vs}_{\mathrm{s}}$ is the systolic velocity in $\mathrm{cm} / \mathrm{s}$ and $\mathrm{Vd}$ is the diastolic velocity in $\mathrm{cm} / \mathrm{s}$. PI was used for the index described by the function: $\mathrm{PI}=\frac{\mathrm{Vs}_{\mathrm{s}} \mathrm{Vd}}{\mathrm{MV}}$. In this function, Vs is the systolic velocity in $\mathrm{cm} / \mathrm{s}, \mathrm{Vd}$ is the diastolic velocity in $\mathrm{cm} / \mathrm{s}$, and $M V$ is the mean velocity in $\mathrm{cm} / \mathrm{s}$. The relevant Doppler parameters used in this review are explained in Table 2 and Fig. 1. Where possible, 95\% confidence intervals of the Doppler-derived parameters were calculated, using sample size, standard deviation, and sample mean.

\section{Assessment of methodological quality}

Three authors (F.C., R.d.G., and F.A.C.) independently assessed the risk of bias. For cohort studies and case-control studies, the appropriate checklist from the Scottish Intercollegiate Guidelines Network (SIGN) was used (http://www.sign.ac.uk/checklists-andnotes.html). Any disagreement was resolved through discussion. Overall quality of the included studies was defined as "low risk of bias," "unclear risk of bias," or "high risk of bias". Decisive items for this overall quality were study group selection, blinding, and correction for confounders.

\section{Statistics}

Because of heterogeneity of Doppler indices, insonated blood vessels, and outcome measures, a quantitative analysis of included studies could not be performed. A narrative summary of the included studies has been provided instead.

\section{RESULTS}

Study selection

The full search identified 7315 articles potentially relevant to our research question, of which 5134 remained after removal of the duplicates. There were no Cochrane reviews meeting our review question. After screening on title and abstract, 57 articles remained. After reading full text, eventually 36 articles met inclusion criteria. One additional study was identified via an expert in the field and one article was identified searching the references of the included studies. Finally, 38 articles were critically appraised, which further led to exclusion of 15 articles based on very high risk of bias, very small

Table 1. Full search strategy in MEDLINE.

\begin{tabular}{|c|c|}
\hline $\begin{array}{l}\text { Time } \\
\text { Items }\end{array}$ & $\begin{array}{l}\text { Full search performed on 08-07-2015 } \\
\text { (Neonate* [tiab] OR Newborn* [tiab] OR Preterm* [tiab] OR Premature* [tiab]) OR “Infant, Newborn"[Mesh] }\end{array}$ \\
\hline & $\begin{array}{l}\text { And }((((((\text { “Echoencephalography"[Mesh]) OR “Ultrasonography, Doppler"[Mesh]) OR "Blood Circulation Time"[Mesh]) OR (((Ultrasound [tiab] OR } \\
\text { US [tiab] OR echo [tiab] OR Doppler [tiab]) AND (Brain [tiab] OR Cerebral [tiab] OR Cranial [tiab])))) OR ((Index [tiab] AND (Resistive [tiab] OR } \\
\text { Resistance [tiab] OR Pourcelot [tiab])))) OR "velocity time" [tiab]) OR velocity OR Echoencephalograp* [tiab] OR Ultrasonogra* [tiab] }\end{array}$ \\
\hline & $\begin{array}{l}\text { and((((cerebral [tiab] OR brain [tiab] OR cranial [tiab]) AND (flow [tiab] OR blood flow [tiab] circulation [tiab])))) OR ((“Brain"[Mesh] AND (“Blood } \\
\text { Vessels" [Mesh] OR "Microcirculation"[Mesh])) OR "Cerebral Arteries"[Mesh] OR “Cerebral Veins"[Mesh] OR "Blood Flow Velocity"[Mesh] OR } \\
\text { "Cerebrovascular Circulation"[Mesh]). }\end{array}$ \\
\hline
\end{tabular}


Table 2. Different Doppler parameters and explanation.

\begin{tabular}{|lll}
\hline Abbreviation & Full term & Equation/explanation \\
\hline RI & Resistance index & Vs - Vd/Vs \\
PI & Pulsatility index & Vs - Vd/MV \\
MV & Mean velocity & Mean velocity calculated over a series of cardiac cycles \\
Vps & Peak-systolic velocity & Highest velocity in the cardiac cycle \\
Ves & End-systolic velocity & Velocity at the end of the systolic phase of cardiac cycle \\
Ved & End-diastolic velocity & Lowest velocity in the cardiac cycle \\
AUVC & Area under the velocity curve & Represents mean flow velocity \\
CBFF & Cerebral blood flow fluctuation & Interquartile range of velocity \\
Min-max MV ratio & Ratio of minimum and maximum MV & Minimal MV/maximal MV \\
Vmean ratio & Ratio of VM at different times & MV in first 12 h/MV at 12-168 h \\
TAV & Time averaged velocity & Vmax/2 \\
CV\% & Coefficient of variability & Coefficient of variation of AUVC values of 20 consecutive \\
BFV & Blood flow velocity & cardiac cycles \\
& Cerebrovascular perfusion pressure & Mean BFV/(mean BFV - diastolic BFV) $\times$ (mean BP - diastolic BP) \\
& Cerebral blood flow resistance & Cerebral perfusion pressure/RI \\
\hline
\end{tabular}

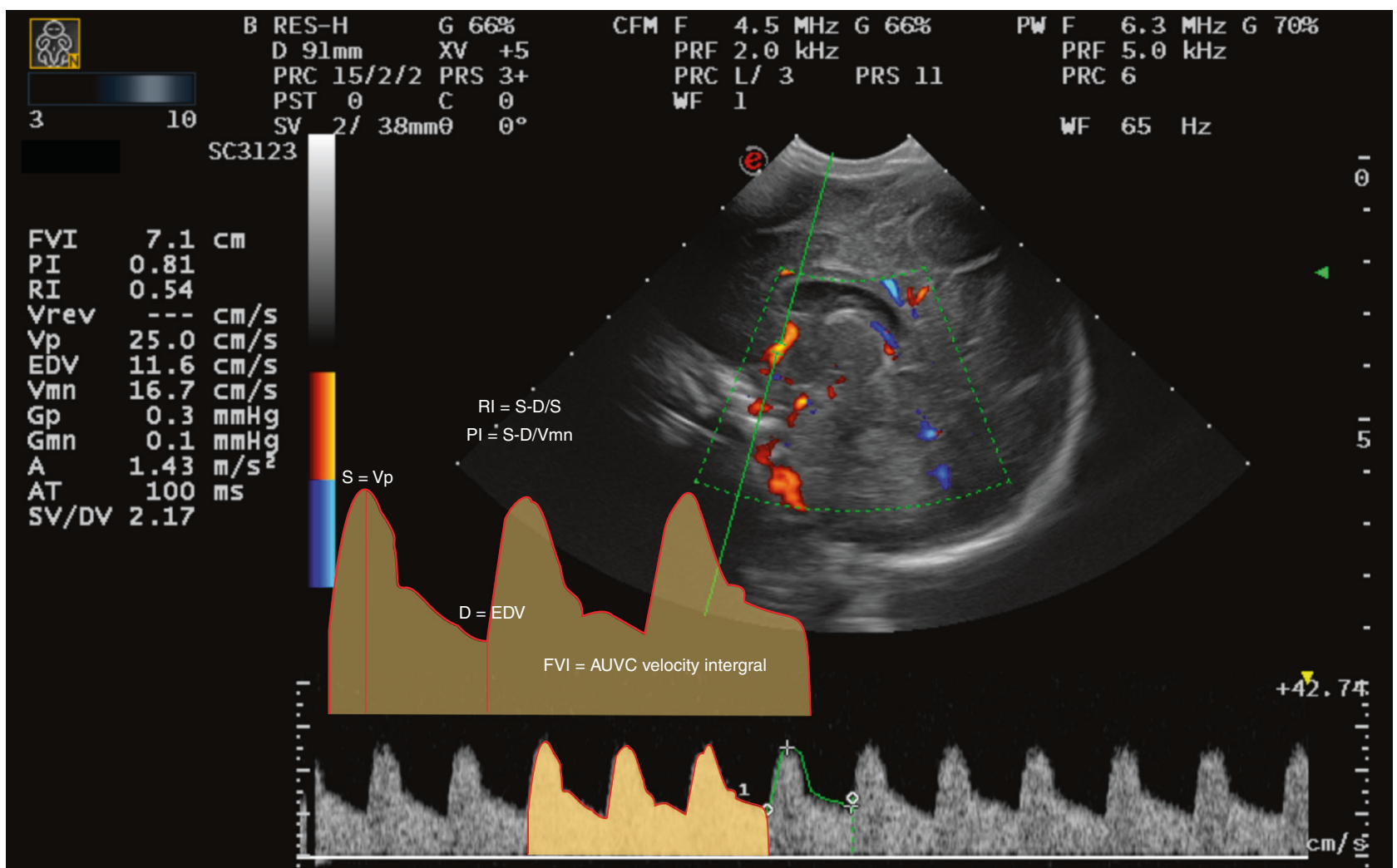

Fig. 1 GMH/IVH: Doppler systematic review: arterial flow indices. AUVC area under the velocity curve, FVI flow velocity integral, Vmn mean velocity $=M V$ in the text.

subgroups ( $<5$ cases per outcome group), or overlapping population with other references. The remaining 23 articles were included in this review (refs.; ${ }^{25-47}$ see Fig. 2 and Appendices 1 and 2).

Study characteristics

In total, 23 studies were included with a total of 2095 preterm participants (median 58 participants per study, range 18-452). Characteristics of the included studies are shown in Appendix 1. In seven older studies, ${ }^{25,27,28,30-33}$ the continuous wave technique with a duplex flowmeter was used to measure Doppler indices. In the remaining 16 studies, real-time two-dimensional (2D) grayscale ultrasonography combined with Color Doppler was applied.

Quality assessment

Out of the 23 included articles, 18 met cohort study design criteria, 2 were considered to be convenience samples with a cohort 


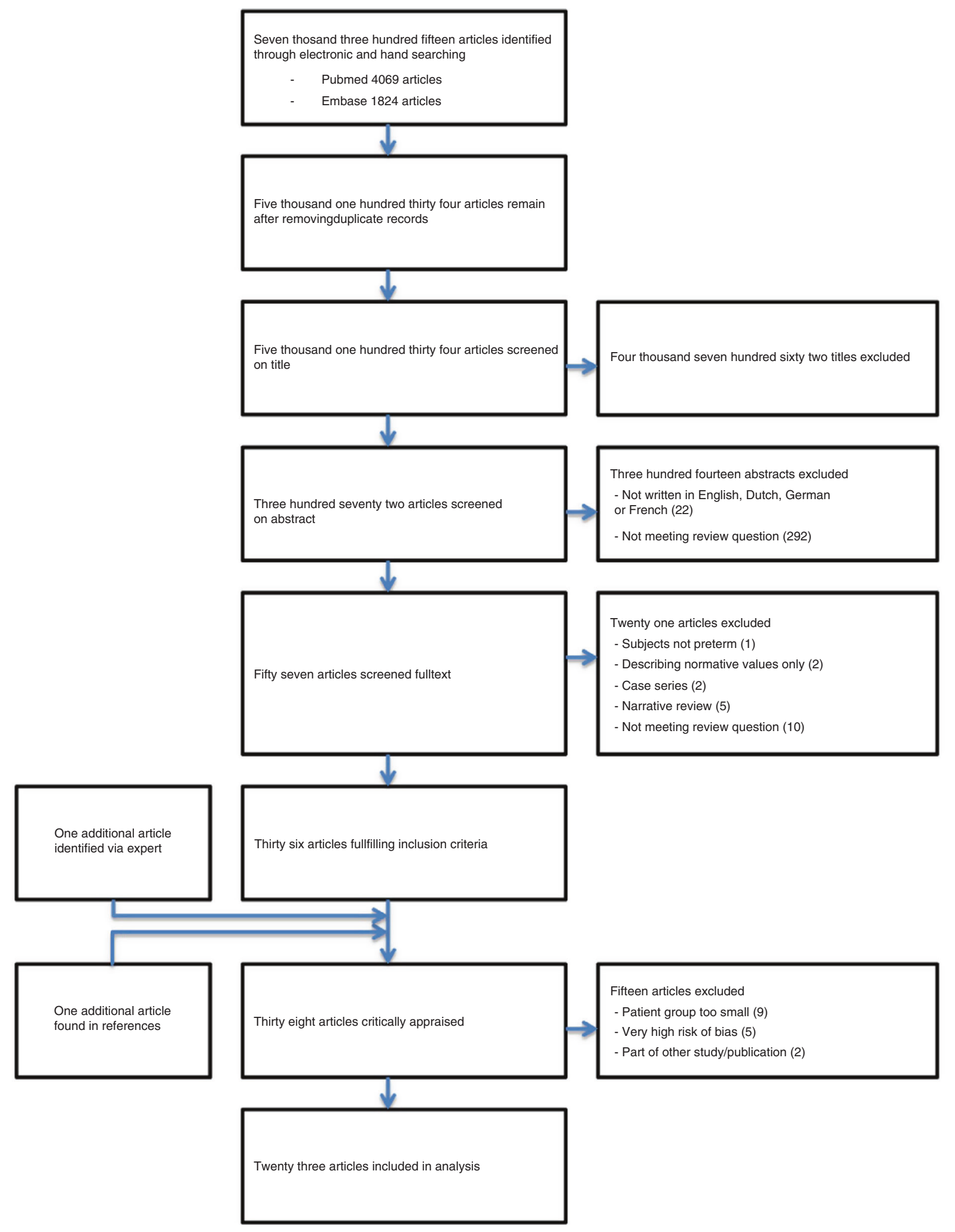

Fig. 2 GMH/IVH: Doppler systematic review.

design, and 3 met case control study criteria. After quality assessment, 7 studies (30\%) were considered to have a low risk of bias, $5(22 \%)$ were considered to have an unclear risk of bias, and $11(48 \%)$ were considered to have a high risk of bias. Considering the separate items of the SIGN checklist, the quality of reporting of the study aim, the definition of outcomes, and the definition of assessment parameters were considered to be good in most of the studies. Overall, criteria for blinding and correction for confounders were less frequently met and therefore a potential risk of bias existed (see Fig. 3). 


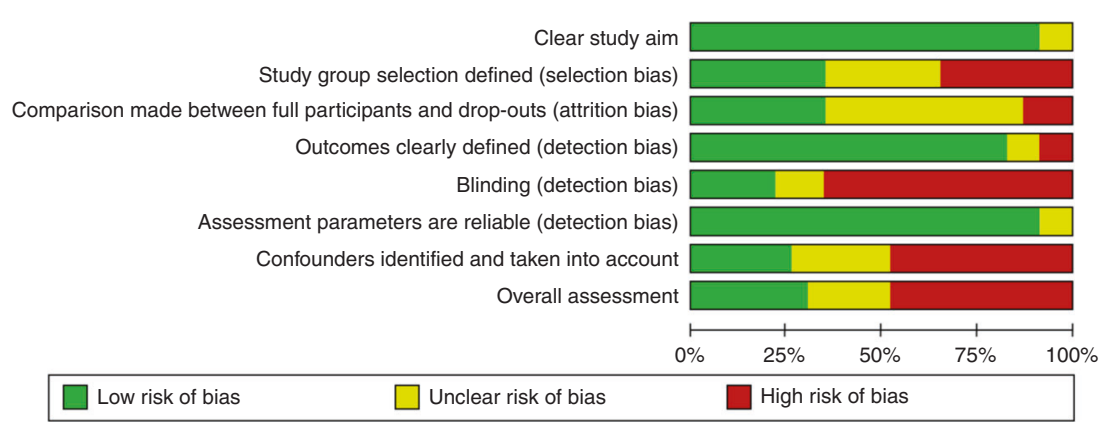

Authors' judgements about each risk of bias item presented as percentages across all included studies. Overall assessment mainly based on study group selection, blinding and correction for confounders
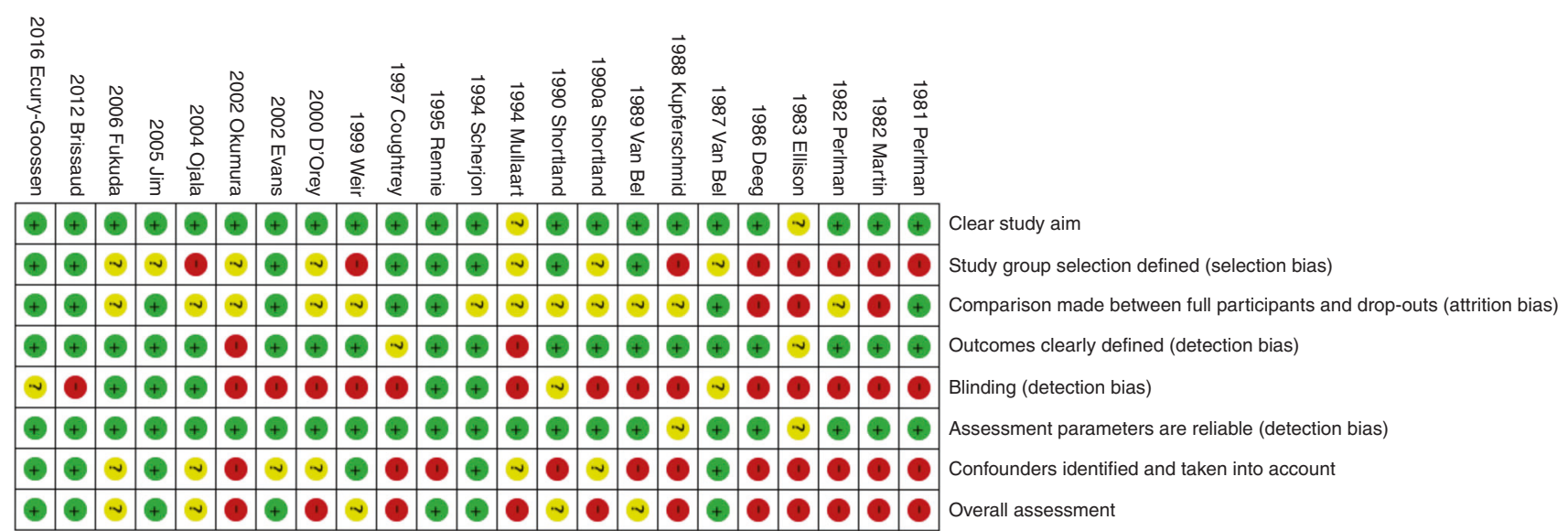

Authors' judgements about each risk of bias item included studies. The item "overall assessment" was mainly based on study group selection, blinding and correction for confounders.

Fig. $3 \mathrm{GMH} / \mathrm{IVH}$ : Doppler systematic review: bias results.

Results of individual studies

In Appendix 2, a summary of the results of each study is presented.

Persistent ductus arteriosus. Eleven studies examined the relationship between cerebral blood flow (CBFV) and PDA. In 9 (82\%) studies, the Doppler parameter to approach CBFV was RI. All studies investigating the $\mathrm{RI}$ in the anterior cerebral artery (ACA8 studies), of which 6 studies considered as high risk of bias and 2 studies considered as low risk of bias, found a significantly higher RI in hemodynamically significant PDA. Mean RI varied between 0.78 and 1.2 in a large PDA compared to RI of $0.61-0.81$ in preterm infants without a significant PDA. ${ }^{25,26,28,29,31,40,44,47}$ Five of these studies used the duplex method without 2D ultrasound. $\mathrm{RI}$ in the middle cerebral artery (MCA) was investigated in two studies, ${ }^{39,40}$ showing a significantly higher RI in patients with PDA. These studies were considered to be moderate and high risk of bias studies, respectively. The internal carotid artery (ICA) was examined in two studies. ${ }^{40,47}$ Both concluded that there was a significantly higher $\mathrm{RI}$ in the ICA in the presence of a hemodynamically significant PDA (median RI 0.82 in PDA versus 0.75 in controls $-p<0.001^{47}$ and mean RI 0.97 in PDA versus 0.81 in controls $-p<0.05^{40}$ ). The $\mathrm{Rl}$ in the common carotid artery (CCA) and in the basilar artery and striatal and pial arteries did not show significant differences in preterm infants with and without hemodynamically significant PDA. ${ }^{28,47}$ Other parameters studied in relation to PDA were coefficient of variation, ${ }^{38} \mathrm{PI}^{39}$ $\mathrm{MV}^{28,34,39,40}$ peak-systolic velocity, end-systolic and end-diastolic velocities, ${ }^{29}$ or maximum and minimum velocities (ratio min-max MV). ${ }^{34}$ For MV, three out of four studies showed significantly lower values in infants with hemodynamically significant PDA. However, absolute MV values differed substantially between those studies (e.g., MV in the ACA ranged from 4.0 to $30.2 \mathrm{~cm} / \mathrm{s}$ in infants with PDA and from 6.5 to $34.9 \mathrm{~cm} / \mathrm{s}$ in controls). Quality assessment of these studies classified two of these studies to be of high risk of bias $^{28,40}$ and one of moderate risk of bias. ${ }^{39}$

Doppler indices and the occurrence of IVH and PVL. In nine studies, a relation between the occurrence of intracranial hemorrhage and/or white matter injury and CBFV was investigated. Six studies examined the relation between CBFV and periventricular hemorrhage, IVH, or peri-intraventricular hemorrhage (PIVH). In five articles (three low risk of bias studies and two high risk of bias studies), no significant correlation was reported using peaksystolic velocity, RI, PI, MV, or cerebral blood flow fluctuation as Doppler parameters for CBFV. ${ }^{33,35,36,41,46}$ One low risk of bias study concluded that RI was significantly lower and the area under the velocity curve (AUVC), RI variation, and AUVC variation in the first week of life were significantly higher in infants who developed severe PIVH. ${ }^{30}$ Two studies explored whether a temporal relation could be detected between an abnormal RI and the onset of IVH. No such relationship could be demonstrated. ${ }^{27,30}$ In five studies, the predictability of PVL by CBFV was assessed. In three of these studies, no correlation was found between CBFV and PVL, using either ACA peak-systolic velocity, ${ }^{46}$ ACA mean velocity, ${ }^{33}$ or MCA mean velocity. ${ }^{36}$ In one study, infants developing PVL had a reduced $\mathrm{Rl}$ in the ACA in the first $72 \mathrm{~h}$ of life, ${ }^{42}$ whereas in another study a lower mean CBFV was not found until beyond the first week of life in the posterior cerebral artery and the ICA and until beyond the second week of life in the ACA, MCA, and basilar artery. $^{45}$

Neurodevelopmental outcome. The relation between neonatal CBFV variables and long-term neurodevelopmental outcome was 
studied in four publications. Different scales were used to evaluate neurodevelopmental outcome.

One moderate risk of bias study showed that low mean CBFV, low CBF resistance (calculated by dividing mean blood pressure by MV as previously described by Evans et al. ${ }^{48}$ ) and low cerebrovascular perfusion pressure (calculated using an equation previously validated by Aaslid et al. ${ }^{49}$ ) in the ACA on the first day of life in non-ventilated infants was associated with a lower Griffith's score at 12 months' corrected age. ${ }^{43}$ A second study, classified as low risk of bias, failed to demonstrate a correlation between the Griffith's scale at 18 months' corrected age and the CBFV in the ACA. ${ }^{37}$ However, infants with an adverse outcome failed to show the steady rise in CBFV during the first days of life, which is observed in infants with normal outcome. One study showed an association between Vmean ratio (MV in the first $12 \mathrm{~h} /$ $\mathrm{MV}$ at $12-168 \mathrm{~h}$ ) and the Prechtl score at term-equivalence but could not demonstrate a correlation between MV or the ratio between the minimal and maximal MV and the Touwen score at 6 and 12 months after birth. ${ }^{36}$ The fourth study, classified as moderate risk of bias, used Griffith's mental developmental scale and Bayley scales of infant development at 2 years' corrected age. $^{32}$ The authors concluded that the RI measured by the duplex flowmeter in the ACA in the first week of life was significantly higher in infants with major impairment.

\section{DISCUSSION}

Twenty-three studies investigating a variety of flow velocity parameters in relation to the presence of a hemodynamically significant PDA, intracranial abnormalities, or neurodevelopmental outcome at 12-18 months' corrected age were identified. Main findings of the studies concerning PDA showed that a hemodynamically significant PDA can be predicted by arterial Doppler patterns. However, there was no consistent evidence that any of the various Doppler-derived variables can predict either development of brain injury or long-term neurodevelopmental impairment.

\section{Persistent ductus arteriosus}

This review found an association between a hemodynamically significant PDA and higher RI and lower MV in the ACA, MCA, and ICA. The presence of a PDA has also been associated with adverse outcome $\mathrm{e}^{50-52}$ and lower cerebral volumes at term-equivalent age. ${ }^{53}$ Altered cerebral blood flow patterns secondary to a PDA, as identified by cerebral Doppler, could therefore help to guide therapeutic strategies. For instance, Bravo et al. ${ }^{54}$ showed that an $\mathrm{RI}$ of $\geq 0.74$ measured $24 \mathrm{~h}$ after termination of ibuprofen treatment was the best biomarker of moderate-to-large PDA (sensitivity $82 \%$ (52-95\%), specificity $72 \%$ (54-84\%), positive predictive value $50 \%(29-71 \%)$, and negative predictive value 92\% (75-98\%). Interestingly, surgical closure of a PDA did not change RI values ${ }^{47}$ and did not improve the long-term neurodevelopmental outcome. ${ }^{55}$ However, in these studies a hemodynamically significant PDA usually is defined only by PDA size, flow pattern, and signs of pulmonary overflow expressed as left atriumto-aorta ratio (LA:Ao). Other echographic signs of systemic repercussion, like low superior vena cava (SVC) flow or absent or reverse flow in any post ductal organ, are usually not taken into account. It might be that a large PDA causes lung overflow but that systemic circulation is not compromised because of compensatory mechanisms.

The high RI and low MV in the ACA is thought to be caused by ductal steal, first described by Spach et al. in $1980 .^{56}$ Ductal steal describes the phenomenon of reduced or even reversed enddiastolic flow to organs secondary to the large ductal shunt toward the lungs during diastole. Since the cerebral circulation in the preterm infant is considered to be a low-resistance system in which diastolic blood flow is an important component, ${ }^{57}$ the preterm brain is thought particularly sensitive to this phenomenon. However, other components must play a role as there is not a consistent relationship between PDA and the long-term outcome, and ductal closure does not consistently lead to normalization of $\mathrm{RI}$ and $\mathrm{MV}^{28,47}$ Interestingly, the RI in the CCA, basilar artery, striatal and pial arteries did not differ among preterm infants with and without a hemodynamically significant PDA. ${ }^{28,47}$ Ecury-Goosen et al. ${ }^{47}$ speculated that the diameter of the insonated artery influences the RI. In the smaller arterial vessels, the diastolic component of the arterial velocity envelope is proportionally higher than in the larger arteries, leading to lower $\mathrm{RI}$ in the first. ${ }^{58}$ This theory only partially explains why in the smaller arteries, such as the striatal and pial arteries, no differences in the RI are found between patients with and without PDA However, one would expect an effect of PDA in the calculated RI in the larger basilar arteries and CCA.

It is important, however, to realize the difference between statistical significance and clinical relevance. The reported values for $\mathrm{Rl}$ in the articles included in this review show normative values ranging from 0.61 to 0.81 , with values associated with a large PDA ranging from 0.78 to 1.2 , therefore showing an overlap. Even in the presence of a hemodynamically significant PDA, the RI is not always consistently altered. One explanation could be that autoregulation is unaltered, ${ }^{59}$ so that the ability to maintain cerebral blood flow stable irrespective of alterations in systemic blood flow remains. Another explanation might be that the RI is not affected when diastolic and systolic flows are equally affected, such as in low cardiac output state. In addition, in some of the included articles, RI values of $>1.0$ were reported as a result of negative diastolic flow. However, accuracy of these values is debatable, since the systolic/diastolic velocity ratio used to calculate RI approaches infinity and therefore loses meaning. ${ }^{60}$ The majority of authors report RI with a range between 0 and 1 as they only use it when the diastolic flow is not reverse.

Doppler indices and the occurrence of IVH and PVL

In this review, we did not find a correlation between the arterial cerebral blood flow Doppler-derived variables and documented structural brain damage (IVH and PVL) during the neonatal period. It is possible that measurements were not done frequently enough. Change in cerebrovascular resistance is a dynamic process, therefore non-continuous assessment may lead to misinterpretation. Van Bel et al. $^{30}$ indeed found a correlation between RI, AUVC, and their variability and the development of IVH during the first week of life. Unfortunately, a temporal relationship between Doppler changes and onset of bleeding could not be demonstrated. ${ }^{27,30}$

Neurodevelopmental outcome

We have not found an association between the Doppler-derived blood flow parameters and long-term neurodevelopmental outcome. However, this could be underpowered for methodological reasons, as the four studies included in this review used neither the same Doppler variables nor the same scale to evaluate neurodevelopmental outcome. The predictive capacity for longterm outcome of arterial Doppler-derived parameters investigated in past decades seems to be weak. Until now, it even remains unclear in which direction the association between Doppler parameters and outcome should be sought. An interesting hypothesis suggested by several authors is that low CBFV is a consequence rather than a cause of brain injury: ${ }^{37,45}$ the usual steady rise in CBFV caused by the increasing metabolic needs of the growing brain ${ }^{61}$ is lacking. The hypothesis is that a damaged brain does not grow as well as a normal brain and therefore demands less blood flow. The fact that lower brain volumes are associated with worse neurodevelopmental outcome supports this theory. ${ }^{62}$ To study predictability for outcome of arterial Doppler parameters, we should focus on studying the same artery 
and the same, standardized scale for neurodevelopmental outcome, which will allow to compare and pool results in future meta-analyses.

\section{General}

In general, many factors are described to influence arterial cerebral blood flow, i.e., maternal smoking, ${ }^{63}$ being small for gestational age, ${ }^{64}$ premature rupture of membranes, ${ }_{1}^{65} \mathrm{pCO}_{2}{ }^{66,67}$ polycythemia, $^{68}$ sedation, ${ }^{69}$ position of the baby, ${ }^{70,71}$ and hypoglycemia. ${ }^{72}$ The role of gestational age is less clear: some studies show a clear correlation with $\mathrm{RI}^{73}$ whereas other studies show no difference. ${ }^{47}$ Many of the babies in the NICU are exposed to more than one of these factors, which may lead to inconsistent results or lack of significance.

Combining arterial cerebral blood flow parameters with clinical parameters or validated clinical scores (e.g., the SNAPPE-II scores) in a model would resolve this problem of significance. Combination of the clinical neurological exam with the existence of lesions on brain MRI or cranial ultrasound improved prediction of neurological outcome in preterm infants. ${ }^{74}$ As far as we know, no such relation was studied between cranial Doppler parameters in preterm babies and clinical parameters.

To the best of our knowledge, this is the first systematic review evaluating diagnostic and predictive value of commonly used cerebral blood flow Doppler-derived measures in relation to both short-term morbidity and longer-term outcome in preterm infants. Studies were published over a long period of time, going back to 1981 , and in general had moderate or high risk of bias. We decided post hoc to exclude 15 studies that met all eligibility criteria because of a very high risk of bias in order to optimize the quality of our findings. One of the strengths of this systematic review is the fact that pre-specified inclusion and exclusion criteria addressing a specific review question were used. In addition, it included a comprehensive search, without limitation of year of publication, limiting risk of selection bias. Finally, formal assessment of risk of bias of included studies was performed allowing to weigh the strength of the evidence.

This review also has several limitations. The inclusion of older studies possibly reduced the applicability of our findings to current clinical practice due to the change in recent decades in the clinical characteristics of the preterm infants in a NICU and to the fact that some Doppler techniques are no longer in use. Also, older studies did not follow the strict standards of reporting that apply today and are therefore more prone to reporting bias. Another limitation of this study is the fact that quantitative analysis was not possible. This was due to important clinical and methodological heterogeneity. Studies varied considerably in terms of technique (duplex versus 2D ultrasound), Doppler parameters, insonated cerebral arteries, timing of measurement, and reporting (use of mean, median, 95\% confidence interval, etc.).

It is possible that we did not find the appropriate Doppler parameter to approach cerebral blood flow yet. RI is often used, since this index shows a very small inter-observer variability and is not dependent on the angle of insonation. Reliability of other Doppler parameters, like end-diastolic, peak-systolic, and mean systolic velocities, is dependent on this angle between the flow velocity and the ultrasound beam. Modern ultrasound machines provide a "steer angle" tool to mathematically correct for this inaccuracy. However, calculations seldom provide true values. To bypass this constrain, using an index that is independent of this angle, like the RI, has become popular to approach CBFV. Greisen et al. ${ }^{75}$ showed that MV might be a better Doppler variable to assess cerebral blood flow patterns than $\mathrm{Rl}$, since the correlation coefficients with CBFV estimated by the Xenon-133 clearance technique were higher for MV than for RI. Unfortunately, in our review $\mathrm{MV}$ also showed a poor performance as predictive tool for IVH $^{41}$ or PVL. ${ }^{33,36}$
An alternative method to approach cerebral circulation using Doppler ultrasound is the SVC flow, ${ }^{76}$ as $70-80 \%$ of the SVC flow represents the cerebral venous return. In several studies, an association between low SVC flow, impaired autoregulation, IVH, and adverse neurodevelopmental outcome was found. ${ }^{14,15,41,77}$ Unfortunately, some authors reported limited reproducibility of SVC flow measurement. ${ }^{78,79}$ However, with appropriate training functional echocardiography is becoming standard of care in many neonatal units in Australia, America, and Europe with reliable and reproducible results. ${ }^{80,81}$

The option of performing venous Doppler measurements instead of arterial ones could be interesting. The venous system is less influenced by vascular tone or shunts (i.e., PDA). With modern ultrasound technique and high-frequency probes, visualization of small vessels and very low flow states, like in the preterm venous circulation, is feasible, provided that the ultrasound probes are calibrated for the specific purpose. ${ }^{82}$ Measurements of changes in perfusion waveform in the internal cerebral vein showed a promising relation with IVH in extremely low birth weight infants. ${ }^{83}$ Future research could focus on the venous system of the preterm brain. Using sufficiently large populations, the same blood vessel at about the same anatomical place in the brain, and preferably in a (semi-)continuous monitoring mode could be a new approach of the cerebral blood flow of the preterm infant.

\section{CONCLUSION}

According to this systematic review, there is no clear evidence to support the routine use of RI or other arterial Doppler-derived parameters to predict neurological outcome in the preterm infant. However, there is some evidence that elevated RI in the ACA and MCA can point to the presence of a hemodynamically significant PDA.

\section{ACKNOWLEDGEMENTS}

The members of eurUS.brain have read and approved this issue. Funding for this publication was provided by the European Society for Paediatric Research (ESPR).

\section{ADDITIONAL INFORMATION}

The online version of this article (https://doi.org/10.1038/s41390-020-0777-x) contains supplementary material, which is available to authorized users.

Competing interests: A.P. has received funding from Chiesi. The authors have declared no competing interests.

Publisher's note Springer Nature remains neutral with regard to jurisdictional claims in published maps and institutional affiliations.

\section{REFERENCES}

1. Back, S. A. Cerebral white and gray matter injury in newborns: new insights into pathophysiology and management. Clin. Perinatol. 41, 1-24 (2014).

2. Meek, J. H., Tyszczuk, L., Elwell, C. E. \& Wyatt, J. S. Low cerebral blood flow is a risk factor for severe intraventricular haemorrhage. Arch. Dis. Child. Fetal Neonatal Ed. 81, F15-F18 (1999).

3. Alderliesten, T. et al. Cerebral oxygenation, extraction, and autoregulation in very preterm infants who develop peri-intraventricular hemorrhage. J. Pediatr. 162, 698-704.e692 (2013).

4. Perlman, J. M., McMenamin, J. B. \& Volpe, J. J. Fluctuating cerebral blood-flow velocity in respiratory-distress syndrome. Relation to the development of intraventricular hemorrhage. N. Engl. J. Med. 309, 204-209 (1983).

5. Perlman, J. M., Goodman, S., Kreusser, K. L. \& Volpe, J. J. Reduction in intraventricular hemorrhage by elimination of fluctuating cerebral blood-flow velocity in preterm infants with respiratory distress syndrome. N. Engl. J. Med. 312, 1353-1357 (1985).

6. Greisen, G. Autoregulation of cerebral blood flow in newborn babies. Early Hum. Dev. 81, 423-428 (2005) 
7. Wong, F. Y. et al. Impaired autoregulation in preterm infants identified by using spatially resolved spectroscopy. Pediatrics 121, e604-e611 (2008).

8. Faust, K. et al. Short-term outcome of very-low-birthweight infants with arterial hypotension in the first $24 \mathrm{~h}$ of life. Arch. Dis. Child. Fetal Neonatal Ed. 100, F388-F392 (2015).

9. da Costa, C. S. et al. Monitoring of cerebrovascular reactivity for determination of optimal blood pressure in preterm infants. J. Pediatr. 167, 86-91 (2015).

10. Waitz, M. et al. Risk factors associated with intraventricular hemorrhage in preterm infants with $\leq 28$ weeks gestational age. Klin. Padiatr. 228, 245-250 (2016).

11. Osborn, D., Paradisis, M. \& Evans, N. The effect of inotropes on morbidity and mortality in preterm infants with low systemic or organ blood flow. Cochrane Database Syst. Rev. CD005090 (2010).

12. Shibasaki, J., Toyoshima, K. \& Kishigami, M. Blood pressure and aEEG in the $96 \mathrm{~h}$ after birth and correlations with neurodevelopmental outcome in extremely preterm infants. Early Hum. Dev. 101, 79-84 (2016).

13. Osborn, D. A., Evans, N. \& Kluckow, M. Hemodynamic and antecedent risk factors of early and late periventricular/intraventricular hemorrhage in premature infants. Pediatrics 112, 33-39 (2003).

14. Riera, J. et al. New time-frequency method for cerebral autoregulation in newborns: predictive capacity for clinical outcomes. J. Pediatr. 165, 897-902 e891 (2014)

15. Riera, J., Cabanas, F., Serrano, J. J., Madero, R. \& Pellicer, A. New developments in cerebral blood flow autoregulation analysis in preterm infants: a mechanistic approach. Pediatr. Res. 79, 460-465 (2016)

16. Raju, T. N. K. Cerebral Doppler studies in the fetus and newborn infant. J. Pediatr. 119, 165-174 (1991).

17. Bada, H. S., Hajjar, W., Chua, C. \& Sumnar, D. S. Noninvasive diagnosis of neonata asphyxia and intraventricular hemorrhage by Doppler ultrasound. J. Pediatr. 95, 775-779 (1979).

18. Vutskits, L. Cerebral blood flow in the neonate. Paediatr. Anaesth. 24, 22-29 (2014).

19. Larsen, F. S., Olsen, K. S., Hansen, B. A., Paulson, O. B. \& Knudsen, G. M. Transcranial Doppler is valid for determination of the lower limit of cerebral blood flow autoregulation. Stroke 25, 1985-1988 (1994).

20. Benders, M. J., Hendrikse, J., de Vries, L., Groenendaal, F. \& van Bel, F. Dopplerassessed cerebral blood flow velocity in the neonate as estimator of global cerebral blood volume flow measured using phase-contrast magnetic resonance angiography. Neonatology 103, 21-26 (2013).

21. Archer, L. N., Levene, M. I. \& Evans, D. H. Cerebral artery Doppler ultrasonography for prediction of outcome after perinatal asphyxia. Lancet 2, 1116-1118 (1986).

22. Hanlo, P. W. et al. Value of transcranial Doppler indices in predicting raised ICP in infantile hydrocephalus. A study with review of the literature. Childs Nerv. Syst. 11, 595-603 (1995).

23. Goh, D. \& Minns, R. A. Intracranial pressure and cerebral arterial flow velocity indices in childhood hydrocephalus: current review. Childs Nerv. Syst. 11, 392-396 (1995).

24. Higgins, J. P. T. \& Green, S. Cochrane Handbook for Systematic Reviews of Interventions (The Cochrane Collaboration, 2011).

25. Perlman, J. M., Hill, A. \& Volpe, J. J. The effect of patent ductus arteriosus on flow velocity in the anterior cerebral arteries: ductal steal in the premature newborn infant. J. Pediatr. 99, 767-771 (1981)

26. Martin, C. G., Snider, A. R., Katz, S. M., Peabody, J. L. \& Brady, J. P. Abnormal cerebral blood flow patterns in preterm infants with a large patent ductus arteriosus. J. Pediatr. 101, 587-593 (1982).

27. Perlman, J. M. \& Volpe, J. J. Cerebral blood flow velocity in relation to intraventricular hemorrhage in the premature newborn infant. J. Pediatr. 100, 956-959 (1982).

28. Ellison, P., Eichorst, D., Rouse, M., Heimler, R. \& Denny, J. Changes in cerebral hemodynamics in preterm infants with and without patent ductus arteriosus. Acta Paediatr. Scand. Suppl. 311, 23-27 (1983).

29. Deeg, K. H. et al. [Doppler sonographic flow parameter of the anterior cerebral artery in patent ductus arteriosus of the newborn infant compared to a healthy control sample]. Klin. Padiatr. 198, 463-470 (1986).

30. Van Bel, F., Van de Bor, M., Stijnen, T., Baan, J. \& Ruys, J. H. Aetiological role of cerebral blood-flow alterations in development and extension of periintraventricular haemorrhage. Dev. Med. Child Neurol. 29, 601-614 (1987).

31. Kupferschmid, C., Lang, D. \& Pohlandt, F. Sensitivity, specificity and predictive value of clinical findings, $\mathrm{m}$-mode echocardiography and continuous-wave Doppler sonography in the diagnosis of symptomatic patent ductus arteriosus in preterm infants. Eur. J. Pediatr. 147, 279-282 (1988).

32. van Bel, F. et al. Cerebral blood-flow velocity during the first week of life of preterm infants and neurodevelopment at two years. Dev. Med. Child Neurol. 31, 320-328 (1989)
33. Shortland, D. B., Levene, M., Archer, N., Shaw, D. \& Evans, D. Cerebral blood flow velocity recordings and the prediction of intracranial haemorrhage and ischaemia. J. Perinat. Med. 18, 411-417 (1990).

34. Shortland, D. B. et al. Patent ductus arteriosus and cerebral circulation in preterm infants. Dev. Med. Child Neurol. 32, 386-393 (1990).

35. Mullaart, R. A. et al. Cerebral blood flow fluctuation in neonatal respiratory distress and periventricular haemorrhage. Early Hum. Dev. 37, 179-185 (1994).

36. Scherjon, S. A., Smolders-DeHaas, H., Oosting, H., Kok, J. H. \& Zondervan, H. A. Neonatal cerebral circulation in relation to neurosonography and neurological outcome: a pulsed Doppler study. Neuropediatrics 25, 208-213 (1994).

37. Rennie, J. M., Coughtrey, H., Morley, R. \& Evans, D. H. Comparison of cerebral blood flow velocity estimation with cranial ultrasound imaging for early prediction of outcome in preterm infants. J. Clin. Ultrasound 23, 27-31 (1995).

38. Coughtrey, H., Rennie, J. M. \& Evans, D. H. Variability in cerebral blood flow velocity: observations over one minute in preterm babies. Early Hum. Dev. 47, 63-70 (1997).

39. Weir, F. J., Ohlsson, A., Myhr, T. L., Fong, K. \& Ryan, M. L. A patent ductus arteriosus is associated with reduced middle cerebral artery blood flow velocity. Eur. J. Pediatr. 158, 484-487 (1999).

40. D'Orey, M. C. et al. Abnormal cerebral flow patterns in preterm infants of less than 33 weeks of gestational age with a large patent ductus arteriosus. Prenat. Neonatal Med. 5, 102-107 (2000).

41. Evans, N., Kluckow, M., Simmons, M. \& Osborn, D. Which to measure, systemic or organ blood flow? Middle cerebral artery and superior vena cava flow in very preterm infants. Arch. Dis. Child. Fetal Neonatal Ed. 87, F181-F184 (2002).

42. Okumura, A. et al. Cerebral hemodynamics during early neonatal period in preterm infants with periventricular leukomalacia. Brain Dev. 24, 693-697 (2002).

43. Ojala, T. et al. Low cerebral blood flow resistance in nonventilated preterm infants predicts poor neurologic outcome. Pediatr. Crit. Care Med. 5, 264-268 (2004).

44. Jim, W. T. et al. Cerebral hemodynamic change and intraventricular hemorrhage in very low birth weight infants with patent ductus arteriosus. Ultrasound Med. Biol. 31, 197-202 (2005).

45. Fukuda, S. et al. Hemodynamics of the cerebral arteries of infants with periventricular leukomalacia. Pediatrics 117, 1-8 (2006).

46. Brissaud, O. et al. Cranial ultrasonography and transfontanellar Doppler in premature neonates (24-32 weeks of gestation): dynamic evolution and association with a severe adverse neurological outcome at hospital discharge in the Aquitaine cohort, 2003-2005. Eur. J. Radiol. 81, 2396-2402 (2012).

47. Ecury-Goossen, G. M. et al. Resistive indices of cerebral arteries in very preterm infants: values throughout stay in the neonatal intensive care unit and impact of patent ductus arteriosus. Pediatr. Radiol. 46, 1291-1300 (2016).

48. Evans, D. H., Levene, M. I., Shortland, D. B. \& Archer, L. N. Resistance index, blood flow velocity, and resistance-area product in the cerebral arteries of very low birth weight infants during the first week of life. Ultrasound Med. Biol. 14 103-110 (1988).

49. Aaslid, R, Lundar, K. F. \& Nornes, H. Estimation of cerebral perfusion pressure from arterial blood pressure and transcranial Doppler recordings. In Proc. Sixth International Symposium on Intracranial Pressure (eds Miller, J. D., Teasdale, G. M., Rowan, J. O., Galbraith, S. L. \& Mendelow, A. D.) 226-229 (Springer-Verlag, Berlin, Heidelberg, 1986).

50. Noori, S. et al. Failure of ductus arteriosus closure is associated with increased mortality in preterm infants. Pediatrics 123, e138-e144 (2009).

51. Heuchan, A. M. \& Clyman, R. I. Managing the patent ductus arteriosus: current treatment options. Arch. Dis. Child. Fetal Neonatal Ed. 99, F431-F436 (2014).

52. Chock, V. Y. et al. Predictors of bronchopulmonary dysplasia or death in premature infants with a patent ductus arteriosus. Pediatr. Res. 75, 570-575 (2014).

53. Lemmers, P. M. et al. Patent ductus arteriosus and brain volume. Pediatrics 137, e20153090 (2016).

54. Bravo, M. C. et al. Randomised controlled clinical trial of standard versus echocardiographically guided ibuprofen treatment for patent ductus arteriosus in preterm infants: a pilot study. J. Matern. Fetal Neonatal Med. 27, 904-909 (2014)

55. Weisz, D. E., More, K., McNamara, P. J. \& Shah, P. S. PDA ligation and health outcomes: a meta-analysis. Pediatrics 133, e1024-e1046 (2014).

56. Spach, M. S., Serwer, G. A., Anderson, P. A. W., Canent, R. V. \& Levin, A. R. Pulsatile aortopulmonary pressure-flow dynamics of patent ductus arteriosus in patients with various hemodynamic states. Circulation 61, 110-122 (1980).

57. Saliba, E. M. et al. Intraoperative measurements of cerebral haemodynamics during ductus arteriosus ligation in preterm infants. Eur. J. Pediatr. 150, 362-365 (1991).

58. Couture, A. \& Veyrac, C. Transfontaneller Doppler Imaging in Neonates (Springer, Berlin, 2001).

59. Chock, V. Y., Ramamoorthy, C. \& Van Meurs, K. P. Cerebral autoregulation in neonates with a hemodynamically significant patent ductus arteriosus. J. Pediatr. 160, 936-942 (2012). 
60. Hendricks, S. K. et al. Doppler umbilical artery waveform indices-Normal values from fourteen to forty-two weeks. Am. J. Obstet. Gynecol. 161, 761-765 (1989).

61. Altman, D. I., Perlman, J. M., Volpe, J. J. \& Powers, W. J. Cerebral oxygen metabolism in newborns. Pediatrics 92, 99-104 (1993).

62. Keunen, K. et al. Brain volumes at term-equivalent age in preterm infants: imaging biomarkers for neurodevelopmental outcome through early school age. J. Pediatr. 172, 88-95 (2016).

63. Abdul-Khaliq, H., Segerer, H., Luck, W. \& Obladen, M. Increased cerebral blood flow velocities in newborn infants of smoking mothers. Eur. J. Pediatr. 152, 232-235 (1993).

64. Milona, E. et al. Evaluation of cerebral perfusion in small for gestational age neonates in the first postnatal week using colour doppler sonography. Arch. Dis. Child. 99, A383-A384 (2014).

65. Fukuda, S. et al. Influence of premature rupture of membrane on the cerebral blood flow in low-birth-weight infant after the delivery. Brain Dev. 32, 631-635 (2010).

66. Fenton, A. C., Woods, K. L., Evans, D. H. \& Levene, M. I. Cerebrovascular carbon dioxide reactivity and failure of autoregulation in preterm infants. Arch. Dis. Child. 67, 835-839 (1992).

67. van Bel, F., van de Bor, M., Baan, J. \& Ruys, J. H. The influence of abnormal blood gases on cerebral blood flow velocity in the preterm newborn. Neuropediatrics 19, 27-32 (1988).

68. D'Orey, M. C. et al. Cerebral blood flow velocimetry in infants with polycythemia: effects of partial exchange transfusion. Prenat. Neonatal Med. 4, 135-138 (1999).

69. Hamon, I., Hascoet, J. M., Debbiche, A. \& Vert, P. Effects of fentanyl administration on general and cerebral haemodynamics in sick newborn infants. Acta Paediatr. 85, 361-365 (1996).

70. Ichihashi, K. et al. Effect of head position to the cerebral arterial flow in neonates. Early Hum. Dev. 69, 35-46 (2002)

71. Buckley, E. M. et al. Cerebral hemodynamics in preterm infants during positional intervention measured with diffuse correlation spectroscopy and transcranial Doppler ultrasound. Opt. Express 17, 12571-12581 (2009).

72. Duckrow, R. B. Decreased cerebral blood flow during acute hyperglycemia. Brain Res. 703, 145-150 (1995).

73. Pezzati, M. et al. Early postnatal Doppler assessment of cerebral blood flow velocity in healthy preterm and term infants. Dev. Med. Child Neurol. 44, 745-752 (2002).

74. Setanen, S. et al. Neurological examination combined with brain MRI or cranial US improves prediction of neurological outcome in preterm infants. Early Hum. Dev. 90, 851-856 (2014).

75. Greisen, G. et al. Cerebral blood flow in the newborn infant: comparison of Doppler ultrasound and 133xenon clearance. J. Pediatr. 104, 411-418 (1984).
76. Kluckow, M. \& Evans, N. Superior vena cava flow in newborn infants: a novel marker of systemic blood flow. Arch. Dis. Child. Fetal Neonatal Ed. 82, F182-F187 (2000).

77. Kluckow, M. \& Evans, N. Low superior vena cava flow and intraventricular haemorrhage in preterm infants. Arch. Dis. Child. Fetal Neonatal Ed. 82, F188-F194 (2000).

78. Ficial, B. et al. Validation study of the accuracy of echocardiographic measurements of systemic blood flow volume in newborn infants. J. Am. Soc. Echocardiogr. 26, 1365-1371 (2013).

79. Lee, A. et al. Superior vena cava flow: feasibility and reliability of the off-line analyses. Arch. Dis. Child. Fetal Neonatal Ed. 95, F121-F125 (2010).

80. McGovern, M. \& Miletin, J. A review of superior vena cava flow measurement in the neonate by functional echocardiography. Acta Paediatr. 106, 22-29 (2017).

81. Mertens, L. et al. Targeted neonatal echocardiography in the neonatal intensive care unit: practice guidelines and recommendations for training. Writing Group of the American Society of Echocardiography (ASE) in collaboration with the European Association of Echocardiography (EAE) and the Association for European Pediatric Cardiologists (AEPC). J. Am. Soc. Echocardiogr. 24, 1057-1078 (2011).

82. Camfferman, F. A. et al. Calibrating Doppler imaging of preterm intracerebral circulation using a microvessel flow phantom. Front. Hum. Neurosci. 8, 1068 (2015).

83. Ikeda, T. et al. Changes in the perfusion waveform of the internal cerebral vein and intraventricular hemorrhage in the acute management of extremely lowbirth-weight infants. Eur. J. Pediatr. 174, 331-338 (2015).

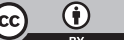

Open Access This article is licensed under a Creative Commons Attribution 4.0 International License, which permits use, sharing, adaptation, distribution and reproduction in any medium or format, as long as you give appropriate credit to the original author(s) and the source, provide a link to the Creative Commons license, and indicate if changes were made. The images or other third party material in this article are included in the article's Creative Commons license, unless indicated otherwise in a credit line to the material. If material is not included in the article's Creative Commons license and your intended use is not permitted by statutory regulation or exceeds the permitted use, you will need to obtain permission directly from the copyright holder. To view a copy of this license, visit http://creativecommons. org/licenses/by/4.0/.

(c) The Author(s) 2020

\section{eurUS.brain group}

Thais Agut ${ }^{8}$, Ana Alarcon ${ }^{8}$, Roberta Arena ${ }^{9}$, Marco Bartocci $^{10}$, Mayka Bravo ${ }^{7}$, Fernando Cabañas ${ }^{11}$, Nuria Carreras ${ }^{8}$, Olivier Claris $^{12}$, Jeroen Dudink ${ }^{3,6}$, Monica Fumagalli ${ }^{13,14}$, Paul Govaert ${ }^{1,3,4,5}$, Sandra Horsch ${ }^{15,16}$, Alessandro Parodi ${ }^{17}$, Adelina Pellicer ${ }^{7}$, Luca Ramenghi $^{17}$, Charles C. Roehr ${ }^{18}$, Sylke Steggerda ${ }^{19}$ and Eva Valverde ${ }^{7}$

${ }^{8}$ Department of Neonatology, Institut de Recerca Pediàtrica, Hospital Sant Joan de Déu, Barcelona, Spain. ${ }^{9}$ Catholic University of the Sacred Heart, A. Gemelli Hospital, Rome, Italy.

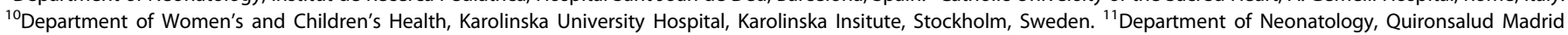
University Hospital and Biomedical Research Foundation, La Paz University Hospital Madrid, Madrid, Spain. ${ }^{12}$ Service de néonatologie et de réanimation néonatale, Hospices Civils

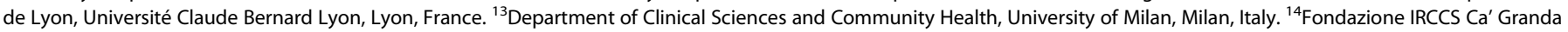

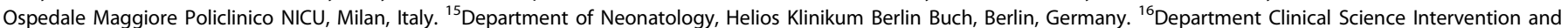

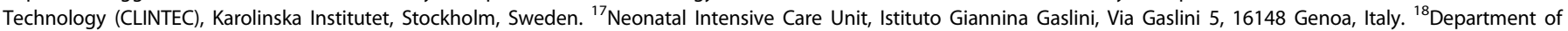

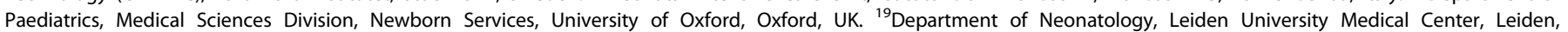
The Netherlands. 